Journal of Engineering and Applied Sciences 14 (21): 7841-7845, 2019

ISSN: 1816-949X

(C) Medwell Journals, 2019

\title{
Sidewall Roughness in Y-Shaped Waveguide: The Effect to the Signal Quality
}

\author{
${ }^{1}$ Mohammad Syuhaimi Ab-Rahman, ${ }^{2}$ I. Shyan Hwang, ${ }^{1}$ Ainon Najahah Abd Aziz and ${ }^{1}$ Foze Ater Saleh \\ ${ }^{1}$ Department of Electric, Electronic and System, \\ Spectrum Technology Research Group (SPECTECH), University Kebangsaan Malaysia, \\ 43600 Bandar Baru Bangi, Malaysia \\ ${ }^{2}$ Department of Computer Engineering and Science, Yuan-Ze University, \\ 135 Yuan-Tung Rd., 32026 Chung-Li, Tao-Yuan, Taiwan
}

\begin{abstract}
In this study the level of sidewall roughness was monitored with depth and structure of roughness to see its effect to signal quality of optical network. Sidewall roughness is contributes to scattering loss in waveguide and leads to signal distortion. Y-shaped waveguide to develop $1 \times 2$ optical splitter was designed with 6 different roughness depth and roughness was placed at 3 different structures. $1 \times 2$ optical splitter waveguide integrated into optical network and the signal quality analyzed by its Q-factor. Simulation results show that Q-factor values was decreasing as roughness get deeper on $0.6 \mu \mathrm{m}$ and sidewall roughness on s-bend structure also, contributes to higher signal distortion compares to roughness on linear structure. Therefore, it is necessary for us to monitor the level of sidewall roughness to ensure the quality of optical signal.
\end{abstract}

Key words: Sidewall roughness, optical splitter, signal quality, optical signal, linear structure, Q-factor

\section{INTRODUCTION}

Optical waveguide is a key element in development of passive optical devices such as optical splitter, switch and multiplexer. The performance of any optical devices highly depends on the waveguide condition. Scattering losses always occur when there is sidewall roughness on the waveguide (Zhao et al., 2004) which sidewall roughness defined as imperfection on vertical surface of waveguide. This contributes to scattering loss, thus, when the optical device from this waveguide installed in any optical network, it cause signal distortion (Kreipe and Unger, 1962). An ideal waveguide condition cannot be realized in practice but we can reduce the level of roughness. The objectives of this study is to see the effect and monitor the level of roughness that can affecting the signal quality and to study the waveguide structure that lead to higher impact.

The imperfection of the waveguide has been studied, since, early 1960 's and numerous theory and experiment on scattering loss due to sidewall roughness has been developed (Kreipe and Unger, 1962; Marcuse, 1969; Lacey and Payne, 1990; Lee et al., 2000). Sidewall roughness of the waveguide can be occur due to some failure during fabrication process that commonly incorporating many process including Reactive Ion Etching (RIE) used to shape the core (Pani et al., 2004; Zhang et al., 2007). The ion bombardment during etching process that causes the roughness throughout the sidewall. Sidewall roughness disturbs the signal transmission guided by the phenomenon of total internal reflection. It scatters the guided waves into unwanted mode and this mode conversion lead to optical loss (Kreipe and Unger, 1962; Van et al., 2001). Optical devices that suffer optical loss will produce low optical power and signal. The quality of signal in the network system will be analyzed using the value of Q-factor. Q-factor is a parameter that directly reflects the quality of a signal of an optical communication system that can be used to monitor the signal quality (Ohteru and Takachio, 1999). The quality of the signal is getting better with the increasing of Q-factor. Quality of signal can be deteriorated by optical noise, nonlinear effects, polarization effects and chromatic dispersion (Shi et al., 2008).

In order to get the smoother sidewall after fabrication process, many techniques was developed (Zhao et al., 2004; Zhang et al., 2007; Wang et al., 2005). After etching process the sidewall condition and its level of roughness can be estimated using Atomic Force Microscopy (AFM) (Di Donato et al., 2010). Since, the

Corresponding Author: Mohammad Syuhaimi Ab-Rahman, Department of Electric, Electronic and System, Spectrum Technology Research Group (SPECTECH), University Kebangsaan Malaysia, 43600 Bandar Baru Bangi, Malaysia 
level of sidewall roughness depends on $\mathrm{O}_{2}$ etching pressure and to resemble this circumstance, in this study, the waveguide was designed with different roughness depths. The position of sidewall roughness also varied to see the effect of structure to signal distortion. The waveguide was designed as a component of $1 \times 2$ optical splitter to be integrated into the optical network.

\section{MATERIALS AND METHODS}

The study of the effect of sidewall roughness was using Y-shaped waveguide as a component of $1 \times 2$ optical splitter. The sidewall roughness of triangle shape was set to 6 different depths from $0 \mu \mathrm{m}$ which is ideal condition, $0.1 \mu \mathrm{m}$ until $0.5 \mu \mathrm{m}$. This depth of roughness determined the level of imperfection for waveguide sidewall which $0 \mu \mathrm{m}$ depth represents low level of roughness while $0.5 \mu \mathrm{m}$ represents the highest level of roughness. Beside the depth of roughness, the effects of structure where sidewall roughness occur was also studied to determine which side or structure of waveguide will contribute to highest loss of optical signal. In order to obtain the result the roughness was placed in three different side linear, The Y-shaped optical waveguide with the sidewall upper s-bend and lower s-bend part as shown in Fig 1. The Y-shaped optical waveguide with the sidewall roughness was designed using waveguide design software, OptiBPM which the waveguides was designed with the addition of triangle shape at their sidewall of the core. The waveguide design was then simulated and integrated into 1.25 Gbps optical network with s ensitivity $-25 \mathrm{dBm}$ prepared using optisystem, network design software as in Fig. 2. For the analysis of signal quality of optical network using $1 \times 2$ optical splitter with sidewall roughness, the Q-factor is the main study parameter.

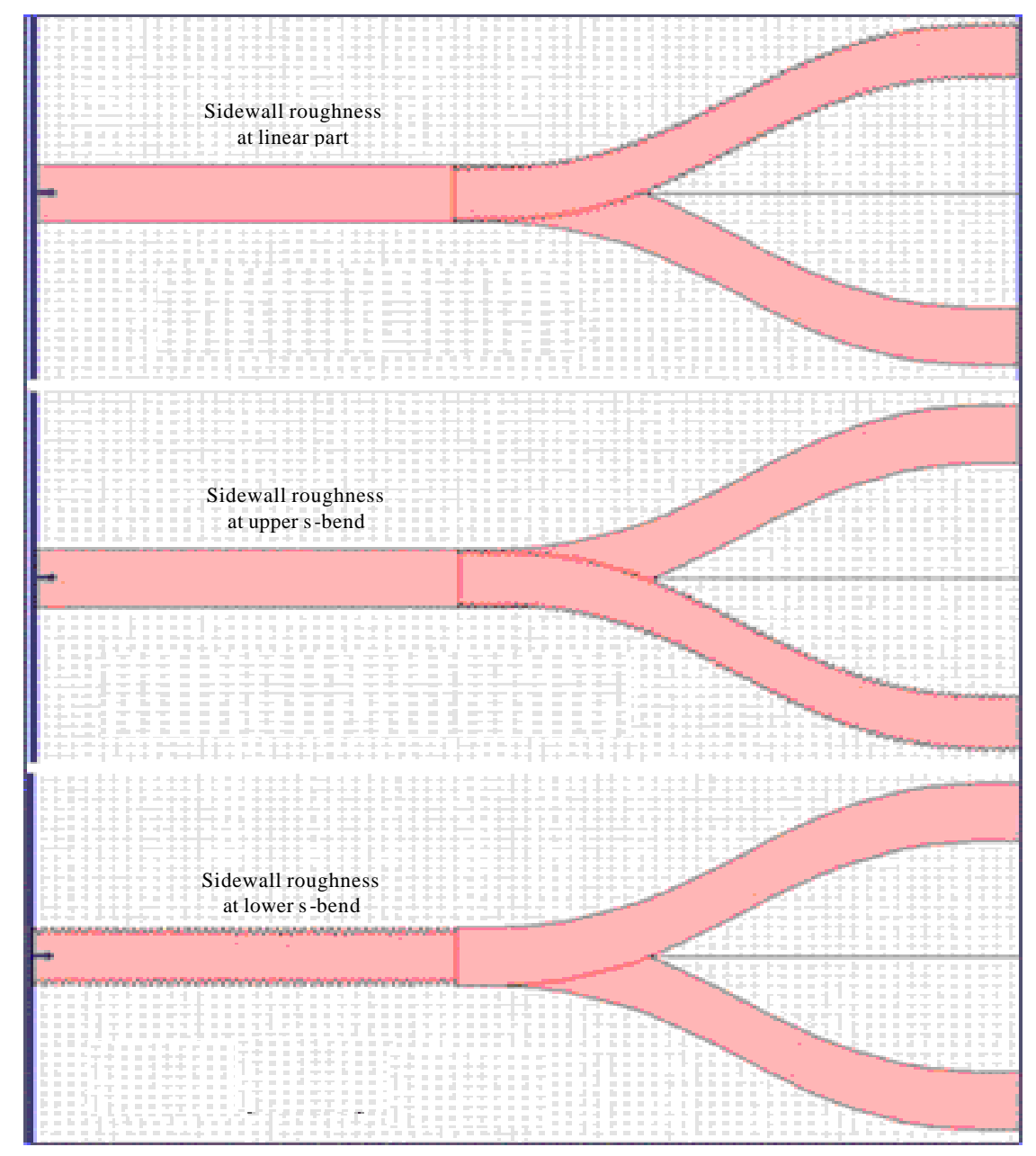

Fig. 1: Different position of triangle roughness in the core sidewall of Y-shaped waveguide 


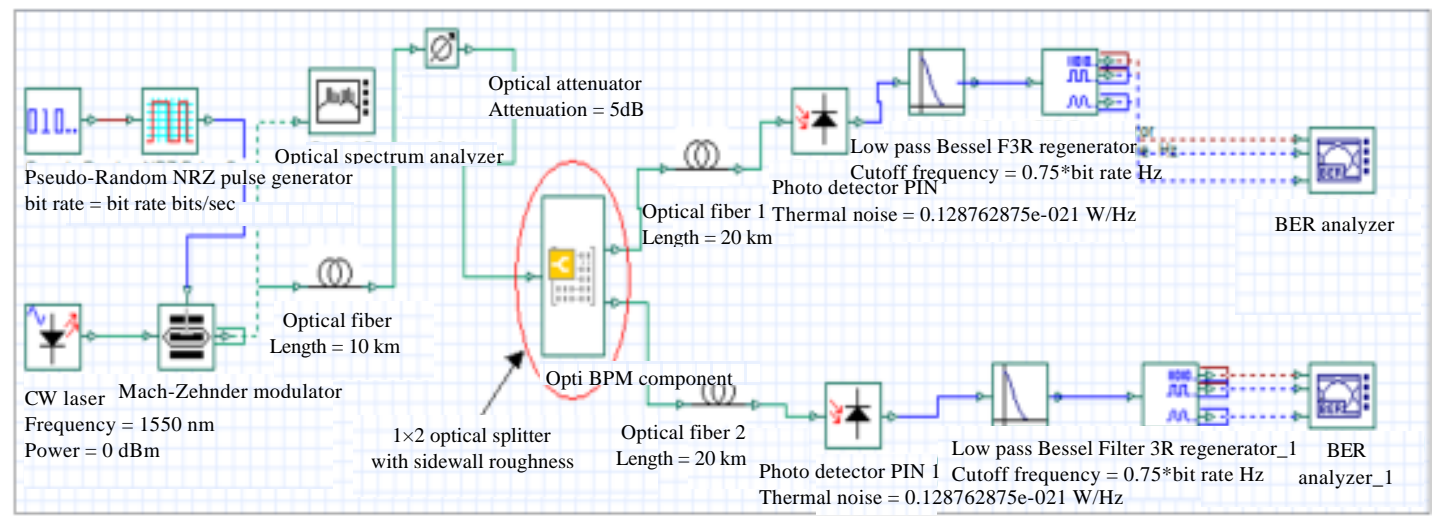

Fig. 2: The integration of $1 \times 2$ optical splitter with rough sidewall waveguide into optical network

\section{RESULTS AND DISCUSSION}

The simulation results to evaluate the signal quality was divided into three parts where the sidewall roughness for $1 \times 2$ optical splitter waveguide was placed at different part and structure linear, upper s-bend and lower s-bend. The value of Q-factor for different roughness depth was taken on both splitter branches that leading to two different Optical Network Units (ONU). For waveguide design with sidewall roughness at linear part, the value of Q-factor from both upper and lower branch decreasing when the roughness get deeper. As depicted in Fig. 3, the value of Q-factor between upper and lower branch have no significant difference and this statement supported by Q-factor value at $0.6 \mu \mathrm{m}$ depth which is 23 for upper branch and 24.1 for lower branch.

This is because after passing through the rough linear part, optical signal was divided equally between both branches. Low level damages with shallow roughness depth might not affect the signal transmission in the waveguide. But when the depth of roughness increasing, transmisson of optical signal through total internal reflection was disturbed which leading to mode conversion and contributes to optical loss. For the results of waveguide with sidewall roughness at upper and lower s-bend, the pattern of increment and decrement of Q-factor value versus the roughness depth is almost the same for both conditions. It can be seen in Fig. 4 and 5 where value of Q-factor for one of the branches would increase while the other one would decrease. The only difference is when roughness occurs on upper s-bend, the value of Q-factor for upper branch decreasing and when roughness occurs on lower s-bend the value of Q-factor for lower branch decreasing.

This is due to the disturbance in propagation of optical signal and loss occurs on the part of damaging waveguide sidewall. While the smooth part of sidewall

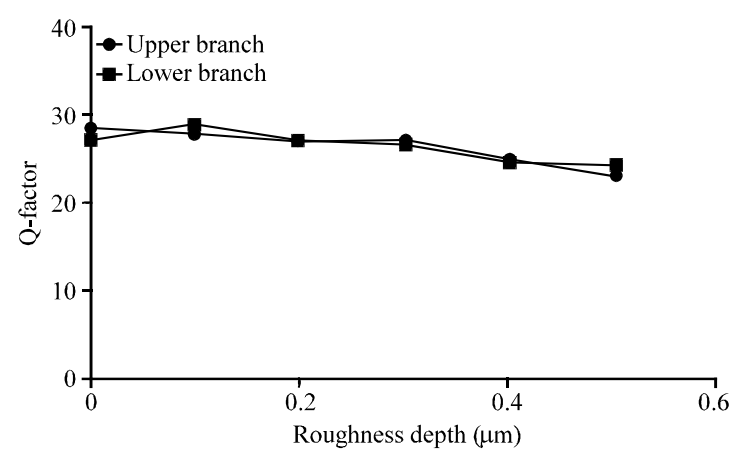

Fig. 3: Roughness depth versus Q-factor for sidewall roughness at linear part

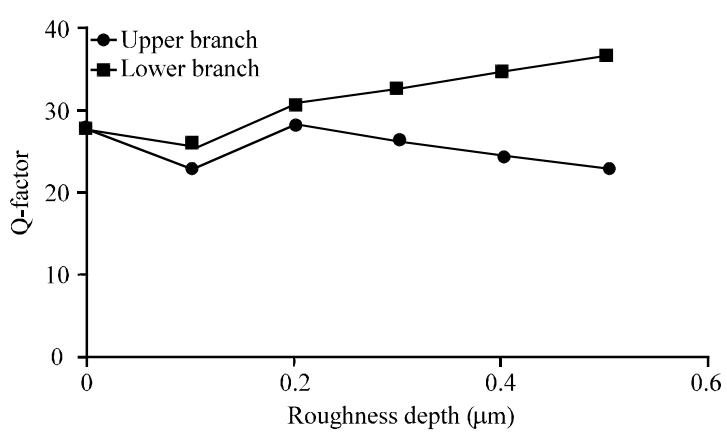

Fig. 4: Roughness depth versus Q-factor for sidewall roughness at upper s-bend

branch will experience the increment of Q-factor value. When the condition of these 2 branches is different, optical power tend to propagates through smoother path compared to rougher path. Thus when the condition between these 2 branches is way the wider gap between total of optical power propagates through them. Optical power is very important, since, it influencing the value of Q-factor and quality of signal. In terms of structure, 


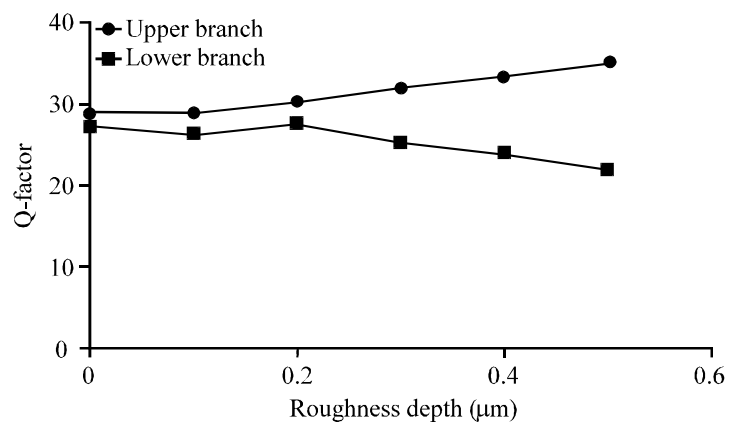

Fig. 5: Roughness depth versus Q-factor for sidewall at lower s-bend

sidewall roughness on s-bend structure contributes to lower signal quality compared to linear structure. Looking into Q-factor values for all the 3 parts, the values just drop to 23 for linear part on $0.6 \mu \mathrm{m}$ depth of roughness while the value of Q-factor on the same depth for upper and lower s-bend drop until 22.9 and 22, respectively. Larger scattering loss expected in s-bend waveguide with larger branching angle (Lee et al., 2000). For network with sensitivity of $-25 \mathrm{dBm}$, roughness depth up to $0.6 \mu \mathrm{m}$ isconsidered acceptable but still should be reduced, since, optical loss not contributed by sidewall roughness only but from many sources as well.

In an effort to improve the quality of signal received by customers, optical loss due to sidewall roughness in any passive optical devices such as $1 \times 2$ optical splitter also need to be reduced. Several researches have been conducted to overcome the problem of sidewall roughness in optical waveguide and should be noted. Refers to the value of Q-factor for different roughness depth it is very important to monitor the sidewall condition of waveguide for any optical device to sustain the quality of signal received by customer in optical network.

\section{CONCLUSION}

Based on the value of Q-factor of different roughness depth it can be concluded that any sidewall roughness on optical waveguide can cause signal distortion. The deeper the roughness the lower the signal quality of optical network analyzed by its Q-factor value. Sidewall roughness on s-bend structure also contributes to higher signal distortion compares to roughness on linear structure. Therefore, it is necessary for us to monitor the level of sidewall roughness to ensure the quality of optical signal and implementing all the techniques to get smooth waveguide sidewall during fabrication process.

\section{ACKNOWLEDGEMENT}

This research activity had been conducted in Computer and Network Security Laboratory, University Kebangsaan Malaysia (UKM). The research was supported by UKM-KK-02-FRGS0195-2010 grant.

\section{REFERENCES}

Di Donato, A., M. Farina, D. Mencarelli, A. Lucesoli and S. Fabiani et al., 2010. Stationary mode distribution and sidewall roughness effects in overmoded optical waveguides. J. Lightwave Technol., 28: 1510-1520.

Kreipe, H.L. and H.G. Unger, 1962. Imperfections in lined waveguide. Bell. Syst. Techn. J., 41: 1589-1619.

Lacey, J.P.R. and F.P. Payne, 1990. Radiation loss from planar waveguides with random wall imperfections. IEE. Proc. J. Optoelectron., 137: 282-288.

Lee, K.K., D.R. Lim, H.C. Luan, A. Agarwal and J. Foresi et al., 2000. Effect of size and roughness on light transmission in a $\mathrm{Si} / \mathrm{SiO} 2$ waveguide: Experiments and model. Appl. Phys. Lett., 77: 1617-1619.

Marcuse, D., 1969. Mode conversion caused by surface imperfections of a dielectric slab waveguide. Bell Syst. Techn. J., 48: 3187-3215.

Ohteru, S. and N. Takachio, 1999. Optical signal quality monitor using direct Q-factor measurement. IEEE. Photonics Technol. Lett., 11: 1307-1309.

Pani, S.K., C.C. Wong, C.S. Premachandran, M.K. Iyer and P.V. Ramana et al., 2004. Pressure and depth dependence of sidewall roughness of polymer optical waveguides during reactive ion etching. Proceedings of 6th International Conference on Electronics Packaging Technology (EPTC 2004) (IEEE Cat. No. 04EX971), December 8-10, 2004, IEEE, Singapore, Singapore, pp: 381-384.

Shi, R., W. Yu, Y. Zhu, C.K. Cheng and E.S. Kuh, 2008. Efficient and accurate eye diagram prediction for high speed signaling. Proceedings of the 2008 IEEE/ACM International Conference on Computer-Aided Design, November 10-13, 2008, IEEE, San Jose, California, USA., ISBN: 978-1-4244-2819-9, pp: 655-661. 
Van, V., P.P. Absil, J.V. Hryniewicz and P.T. Ho, 2001. Propagation loss in single-mode GaAs-AlGaAs microring resonators: Measurement and model. J. Lightwave Technol., 19: 1734-1739.

Wang, Z., B. Yang, Z. Fu, W. Dong and Y. Yang et al., 2005. UV-blue photoluminescence from $\mathrm{ZrO}_{2}$ nanopowders prepared via glycine nitrate process. Appl. Phys. A., 81: 691-694.
Zhang, H.M., C.S. Ma, Z.K. Qin, X.Z. Zhang and S.Y. Liu et al., 2007. Reduction of sidewall roughness, insertion loss and crosstalk of polymer arrayed waveguide grating using vapor-redissolution technique. Thin Solid Films, 515: 7313-7317.

Zhao, Y., D.M. Zhang, F. Wang, Z.C. Cui and M.B. Yi et al., 2004. Fabrication techniques for colymer/Si optical waveguide. Opt. Laser Technol., 36: $657-660$. 